\title{
Complex-scaling Fourier-grid Hamiltonian method. III. Oscillatory behavior of complex quasienergies and the stability of negative ions in very intense laser fields
}

\author{
Guanhua Yao and Shih-I Chu \\ Department of Chemistry, University of Kansas, Lawrence, Kansas 66045 \\ (Received 8 October 1991; revised manuscript received 5 December 1991)
}

\begin{abstract}
A complex-scaling Fourier-grid Hamiltonian (CSFGH) method in momentum representation along with Floquet formalism is presented and applied to the nonperturbative treatment of the complex quasienergies of a one-dimensional-model negative chlorine ion in an ArF excimer-laser field. The CSFGH method leads to a simple and highly efficient variational procedure for accurate determination of resonance states, without the need of using an $L^{2}$-basis-function expansion. A variety of laser intensities are considered covering those from perturbative to very intense laser fields, and the stability of the ion is investigated under realistic experimental conditions. Oscillatory structures of the photodetachment rate as a function of field strength are found in our study, revealing that the decreasing of the detachment rate (or the so-called "stabilization" or "suppression-of-ionization" phenomenon) may not necessarily be a monotonic behavior. Physical insight into this interesting feature is explored in the Kramers-Henneberger frame, and the feasibility for the experimental observation of the oscillatory behavior of the negative ions is also discussed.
\end{abstract}

PACS number(s): 32.80.Rm, 32.80.Wr, 31.15. + q, 32.70.Jz

\section{INTRODUCTION}

According to experimental observations and most theoretical calculations, the lifetime of an atom in a strong laser field would decrease with increasing laser intensity. However, recent theoretical studies on photoionization of atoms in high-frequency very intense fields have found features contrary to the common expectations, and have thus attracted considerable interest in this new domain. Notably, the decay rate, or the ionization probability of the atom, is predicted to decrease with increasing intensity, and the atom becomes relatively stable against ionization (so-called "suppression of ionization") $[1,3,4]$.

Several theoretical approaches have been used to study the very intense laser-atom interaction. Pont, Gavrila, and co-workers [1] considered the high-frequency limit by making the Kramers-Henneberger ( $\mathrm{KH}$ ) transformation [2] and by keeping the zeroth-order term. In this asymptotic regime, they showed that the atomic electron simply moved in a time-independent field-deformed potential and was stabilized. In their later publications [3], they extended their studies by including higher-order channels. By approximately relating the square of the coupling matrix element between field-deformed ground and continuum states to the decay rate, they observed the stabilization of hydrogen atoms in the high-field highfrequency limit. In addition, time-dependent methods have been employed in the numerical solution of the Schrödinger equation [4]. Along this line of work, the ionization probability has been calculated and suppression of ionization has also been demonstrated for veryhigh-field intensities. Few exact rate calculations, however, have been carried out for the very-intense-field regime.
As has been indicated [3], the high-frequency approximation leading to stabilization requires that the laser frequency $\omega \gg\left|E_{0}\right|$ (the field-deformed ground-state energy). Thus stabilization of the hydrogen atom requires unrealistically high frequencies. Actually almost all the calculations relating to the problem have been performed for $\omega$ larger than 0.5 a.u. and for laser intensities in excess of 1 a.u. at those frequencies $[1,3,4]$, which are well beyond realistic expectations for the foreseeable future. Furthermore, the pulse length, and especially the pulserising time, needed for obtaining suppression are exclusively short (typically a few femtoseconds and subfemtoseconds, respectively) in order for the neutral atom to survive up to the peak intensity and to experience suppression. In particular, according to Pont and Gavrila's calculations [3], the minimum lifetime of hydrogen atoms is about 0.1 fs (or one optical cycle) for $\omega=1.0$ a.u. The rising time must be even shorter in order to survive the atom. As a result, attempts to observe the suppression of ionization will require the use of currently unavailable high-intensity $x$-ray lasers. Tang and Basile [4(a)] reached the same conclusion through their time-dependent calculations. It seems that stabilization of hydrogen atoms in very intense fields cannot be realized in current laboratory conditions, at least for the ground state. The situation may be different for excited states [1(b),5].

In comparison, atomic negative ions possess small binding energies and short-range potentials. The highfrequency condition for such ions requires only moderate (at most uv) frequency and intensity (less than one atomic unit in our case) which are fairly available in view of the modern state of the art of excimer-laser technologies [6]. It is therefore one of our main purposes in the present pa- 
per to study the nonlinear response of negative ions in very intense laser fields and to seek the conditions for ionization suppression suitable for experimental observation. The second motivation relies on the fact that accurate determination of multiphoton-ionization rates is usually difficult to perform in the very-intense-field regime. Development of practical and efficient procedures is thus urgent in order to explore new atomic physics in the veryintense-field regime.

In this paper, a complex-scaling Fourier-grid Hamiltonian (CSFGH) method in momentum representation will be presented and applied to the nonperturbative calculation of the multiphoton-detachment rate for a model negative chlorine ion in intense fields. Oscillatory structures and stabilization are found and the feasibility for experimental observations will be explored. The method is presented in Sec. II and applied to a model $\mathrm{Cl}^{-}$ion in Sec. III. In Sec. IV, the origin of the oscillatory structures in photodetachment rates is explored. This is followed by a conclusion in Sec. V.

\section{METHODS}

\section{A. Fourier-grid Hamiltonian method in momentum representation}

Recently, one of us has developed a complex-scaling Fourier-grid Hamiltonian method in coordinate representation $[7,8]$ for accurate and efficient determination of resonance energies and wave functions of metastable states. The CSFGH method makes use of the recent advancement of the calculation of bound states using the Fourier transformation method [9]. The procedure does not involve the use of $L^{2}$-basis-function expansion and no computation of potential matrix elements (which is usually the most time consuming part) is required. Because of its extreme simplicity in numerical implementation, the method has decisive advantages for problems where no appropriate basis set can be found or a large number of basis functions are required. The CSFGH method has been recently applied successfully to the calculation of resonance states of an anharmonic oscillator [7] (hereafter referred to as I) and to the study of multiphoton and above-threshold dissociation of $\mathrm{H}_{2}{ }^{+}$in intense laser fields [8] (hereafter referred to as II). In the latter study, 51 grid points are found to be sufficient to achieve at least four significant digits of accuracy for the complex quasienergies $\left(E_{R},-\Gamma / 2\right)$ of both low-lying and highly excited vibrational states $(v=0,1,2, \ldots, 16)$ of $\mathrm{H}_{2}{ }^{+}\left(1 s \sigma_{g}\right)$ electronic state.

In this section, we discuss the extension of the Fourier-grid Hamiltonian (FGH) method to the momentum representation. As will be shown below, for certain problems (particularly those involving short-range potentials) there are additional advantages for discretizing the Hamiltonian operator in the momentum $(k)$ space. The advantages of working in momentum representation have also been noticed by other authors in different contexts or applications [10].

For simplicity, let us consider first the one-dimensional (1D) problem with the Hamiltonian

$$
\hat{H}=\widehat{T}+\hat{V}(\hat{x})=\hat{p}^{2} / 2 m+\hat{V}(\hat{x}) .
$$

It is known that the kinetic-energy operator $\widehat{T}$ is diagonal in momentum representation,

$$
\left\langle k^{\prime}|\hat{T}| k\right\rangle=\left(\hbar^{2} k^{2} / 2 m\right) \delta\left(k-k^{\prime}\right),
$$

while the potential-energy term $\hat{V}(\hat{x})$ is diagonal in the coordinate representation,

$$
\left\langle x^{\prime}|\hat{V}(\hat{x})| x\right\rangle=V(x) \delta\left(x-x^{\prime}\right) .
$$

In the coordinate or Schrödinger representation, the Hamiltonian operator in Eq. (1) becomes

$$
\left\langle x|\hat{H}| x^{\prime}\right\rangle=\left\langle x|\hat{T}| x^{\prime}\right\rangle+V(x) \delta\left(x-x^{\prime}\right) .
$$

Similarly, in the momentum representation, we have

$$
\left\langle k|\hat{H}| k^{\prime}\right\rangle=\left(\hbar^{2} k^{2} / 2 m\right) \delta\left(k-k^{\prime}\right)+\left\langle k|\hat{V}(\hat{x})| k^{\prime}\right\rangle .
$$

As shown in papers I and II, it is generally advantageous to work in the coordinate representation Eq. (4) since the potential $\hat{V}(\hat{x})$ is diagonal and no potential matrix elements need to be computed. However, for certain problems such as negative ions where $V(\hat{x})$ is of short range, the momentum representation offers several advantages. First, the kinetic-energy operator is diagonal. Second, the potential matrix element

$$
V\left(k^{\prime}-k\right) \equiv\left\langle k|\hat{V}(\hat{x})| k^{\prime}\right\rangle=\left(\frac{1}{2} \pi\right) \int_{-\infty}^{\infty} d x V(x) e^{i\left(k^{\prime}-k\right) x}
$$

is simply the Fourier transform of $V(x)$ and can often be worked out in a simple analytic form and evaluated efficiently. Third, for certain potentials $V(x)$ such as the square-well type or potentials with a cusp, discretization in $x$ space with equal spacing [9] would be very inefficient. In such circumstances, the momentum representation would be of advantage since the Fourier transformation of $V(x)$ Eq. (6) is often in smoother and of slowly varying form in $k$ space.

We now consider the discretization of the Hamiltonian in momentum representation Eq. (5). Here a uniform discrete grid point (with spacing $\Delta k$ ) in $k$ space, evenly distributed about $k=0$, will be used. Thus, the discretized Hamiltonian in $k$ space has the form

$H_{i j}=\left\langle k_{i}|\hat{H}| k_{j}\right\rangle=\left(\hbar^{2} k_{i}^{2} / 2 m\right) \delta_{i j} / \Delta k+V\left(k_{i}-k_{j}\right)$.

This leads to the following secular equation:

$$
\sum_{j}\left(H_{i j}-W_{v} \delta_{i j}\right) \psi_{j}^{v}=0,
$$

where the eigenvectors $\psi_{j}^{v}$ provide directly the amplitude of the normalized wave function evaluated at the grid point $k_{j}$, namely,

$$
\psi_{j}^{v}=\left\langle k_{j} \mid \psi^{v}\right\rangle=\psi^{v}\left(k_{j}\right) .
$$

If the wave function in coordinate representation is needed, it can be obtained by a simple Fourier transform, viz.,

$$
\psi^{v}(x)=\left\langle x \mid \psi^{v}\right\rangle=\frac{1}{\sqrt{2 \pi}} \int_{-\infty}^{\infty} d k \psi^{v}(k) e^{i k x},
$$

or written in the discretized form 


$$
\psi^{v}\left(x_{i}\right)=\left\langle x_{i} \mid \psi^{v}\right\rangle=\frac{\Delta k}{\sqrt{2 \pi}} \sum_{j} \psi^{v}\left(k_{j}\right) \exp \left(i k_{j} x_{i}\right) .
$$

As an example of the usefulness of the momentum representation FGH method in calculating the bound-state energies, let us consider the following Gaussian-type model potential for describing the short-range $\mathrm{Cl}^{-}$ion:

$$
V(x)=-V_{0} \exp \left[-\left(x / x_{0}\right)^{2}\right],
$$

with $V_{0}=0.27035$ a.u. and $x_{0}=2.0$ a.u. (This potential form has been used recently by Bardsley and Comella [11] for describing model neutral atoms with a few bound states.) This potential in momentum representation has a simple analytic form,

$V\left(k^{\prime}-k\right)=-\left(V_{0} x_{0} / 2 \sqrt{\pi}\right) \exp \left[-\left(k^{\prime}-k\right)^{2} x_{0}^{2} / 4\right]$.

The potential Eq. (12) or Eq. (13) supports only one bound state. The binding energy of the model $\mathrm{Cl}^{-}$ion calculated by the momentum representation FGH method [using 81 grid points (evenly distributed between $-k_{\max } / 2$ and $\left.+k_{\max } / 2\right)$ ] converges to at least $10^{-10}$ a.u., indicating the method is stable and accurate.

\section{B. Complex-scaling Fourier-grid Hamiltonian method in momentum representation: Determination of complex quasienergies in the Kramers-Henneberger frame}

In this section, we discuss the extension of the complex-scaling Fourier-grid Hamiltonian methods [7,8] to momentum representation for nonperturbative treatment of complex quasienergies associated with multiphoton detachment of negative ions. We assume the quantum systems are driven by monochromatic fields with the Hamiltonian periodic in time. [Extension to photodetachment in multicolor (polychromatic) fields can be achieved by means of the many-mode Floquet theory $[12,13]$, and will be discussed elsewhere.] In the presence of very intense laser fields, it has been pointed out by several authors that the velocity gauge is more appropriate and superior to the length gauge $[4,8]$. As the interaction operator in the velocity gauge is diagonal in momentum representation, it is expedient to cast the time-dependent Schrödinger equation into the $k$ space. Furthermore, the Kramers-Henneberger transformed version of the Schrödinger equation is in more transparent form in this representation, as we will see below.

The Schrödinger equation of an atomic system in an electromagnetic field (in velocity gauge) is $(\hbar=1)$

$$
i \frac{\partial}{\partial t} \psi(\mathbf{r}, t)=\left\{[\hat{\mathbf{p}}-e \mathbf{A}(t)]^{2} / 2 m+\hat{V}(\mathbf{r})\right\} \psi(\mathbf{r}, t) .
$$

The $k$-space version of this equation is

$$
\begin{aligned}
i \frac{\partial}{\partial t} \widetilde{\phi}(\mathbf{k}, t)= & \left\{[\mathbf{k}-e \mathbf{A}(t)]^{2} / 2 m\right\} \widetilde{\phi}(\mathbf{k}, t) \\
& +\int d \mathbf{k}^{\prime} V\left(\mathbf{k}^{\prime}-\mathbf{k}\right) \widetilde{\phi}\left(k^{\prime}, t\right),
\end{aligned}
$$

where $V\left(\mathbf{k}^{\prime}-\mathbf{k}\right)$ is the field-free potential in $\mathbf{k}$ space [cf. Eq. (6)], and $\widetilde{\phi}(\mathbf{k}, t)$ is the time-dependent wave function in $\mathbf{k}$ space.

It is useful to introduce a new unitarily transformed wave function:

$$
\phi(\mathbf{k}, t)=\widetilde{\phi}(\mathbf{k}, t) e^{i \Pi(t)} e^{-i \mathbf{k} \cdot \boldsymbol{\alpha}(t)},
$$

where

$$
\Pi(t)=\int_{-\infty}^{t} d \tau e^{2} A^{2}(\tau) / 2 m
$$

and

$$
\alpha(t)=(e / m) \int_{-\infty}^{t} d \tau \mathbf{A}(\tau) .
$$

The Schrödinger equation for $\phi(\mathbf{k}, t)$ now becomes

$$
\begin{aligned}
i \frac{\partial}{\partial t} \phi(\mathbf{k}, t)= & \frac{k^{2}}{2 m} \phi(\mathbf{k}, t) \\
& +\int d \mathbf{k}^{\prime} V\left(\mathbf{k}^{\prime}-\mathbf{k}\right) e^{i\left(\mathbf{k}^{\prime}-\mathbf{k}\right) \cdot \boldsymbol{\alpha}(t)} \phi\left(\mathbf{k}^{\prime}, t\right) .
\end{aligned}
$$

If we transform this equation back to the coordinate space, it is exactly the KH-transformed Schrödinger equation in coordinate space, with

$$
V(\mathbf{r}+\boldsymbol{\alpha}(t))=\int d \mathbf{k} V(\mathbf{k}) e^{-i \mathbf{k} \cdot \boldsymbol{\alpha}(t)} e^{-i \mathbf{k} \cdot \mathbf{r}} .
$$

As a result, Eq. (16) is simply the counterpart of $\mathrm{KH}$ transformation in momentum representation.

For the periodic laser field $\mathbf{A}(t)=\mathbf{A}_{0} \cos \omega t$ we have

$$
\begin{aligned}
\boldsymbol{\alpha}(t)=\alpha_{0} \sin \omega t & =\left(e \mathbf{A}_{0} / m \omega\right) \sin \omega t \\
& =\left(e \mathbf{E}_{0} / m \omega^{2}\right) \sin \omega t
\end{aligned}
$$

Using the Fourier expansion, Eq. (19) becomes

$$
\begin{aligned}
i \frac{\partial}{\partial t} \phi(\mathbf{k}, t)= & \frac{k^{2}}{2 m} \phi(\mathbf{k}, t)+\int d \mathbf{k}^{\prime} V_{0}\left(\mathbf{k}^{\prime}-\mathbf{k}\right) \phi\left(\mathbf{k}^{\prime}, t\right) \\
& +\sum_{n \neq 0} e^{i n \omega t} \int d \mathbf{k}^{\prime} V_{n}\left(\mathbf{k}^{\prime}-\mathbf{k}\right) \phi\left(\mathbf{k}^{\prime}, t\right)
\end{aligned}
$$

where

$$
V_{n}(\mathbf{k})=V(\mathbf{k}) J_{n}\left(\alpha_{0} \cdot \mathbf{k}\right),
$$

and the $J_{n}$ 's are the Bessel functions.

In the high-frequency limit, the $n \neq 0$ terms in Eq. (22) oscillate very rapidly and in the zeroth-order approximation, they can be neglected. This reduces Eq. (22) to the time-independent zeroth-order $(n=0)$ equation which possesses only bound-state solutions. The properties of these field-deformed bound eigenstates have been discussed extensively in the literature [1].

In the following, we shall discuss the solution of Eq. (22) for the determination of complex quasienergies of a model negative ion driven by very intense fields. To this end, we introduce the complex-scaling transformation [13] $\mathbf{k} \rightarrow \mathbf{k} e^{-i \theta}$ in Eq. (22) and discretize the corresponding Hamiltonian $\hat{H}(\theta)$ in $\mathbf{k}$ space. For simplicity, we shall confine our discussions to the 1D case below.

First, notice the complex-scaled Hamiltonian matrix element has the following form in $k$ space:

$$
\begin{aligned}
\left\langle k|\hat{H}(\theta)| k^{\prime}\right\rangle= & \frac{k^{2}}{2 m} e^{-2 i \theta} \delta\left(k-k^{\prime}\right) \\
& +e^{-i \theta} \sum_{n=-\infty}^{\infty} e^{i n \omega t} V_{n}\left(\left(k^{\prime}-k\right) e^{-i \theta}\right)
\end{aligned}
$$


This periodically time-dependent Hamiltonian can be transformed into an equivalent infinite-dimensional time-independent non-Hermitian Floquet Hamiltonian $\hat{H}_{F}(\theta)$ [13]. In terms of the Floquet state basis $|k n\rangle \equiv|k\rangle_{\otimes}|n\rangle$, the matrix elements of $\hat{H}_{F}$ are given by $(\hbar=1)$

$$
\begin{aligned}
\left\langle k n_{1}\left|\hat{H}_{F}(\theta)\right| k^{\prime} n_{2}\right\rangle= & {\left[\frac{k^{2}}{2 m} e^{-2 i \theta}+n_{1} \omega\right] \delta\left(k-k^{\prime}\right) \delta_{n_{1} n_{2}} } \\
& +e^{-i \theta} V_{n_{1}-n_{2}}\left(\left(k^{\prime}-k\right) e^{-i \theta}\right)
\end{aligned}
$$

where $n_{1}$ and $n_{2}$ are the Fourier indices $(-\infty$ to $+\infty)$ and $V_{n}(k)$ is defined by Eq. (23). Now we further discretize the $k$ space as described in Sec. II A, yielding the following discretized non-Hermitian Floquet Hamiltonian:

$$
\begin{aligned}
\left\langle k_{i} n_{1}\left|\hat{H}_{F}(\theta)\right| k_{j} n_{2}\right\rangle= & (1 / \Delta k)\left[\frac{k_{i}^{2}}{2 m} e^{-2 i \theta}+n_{1} \omega\right] \delta_{i j} \delta_{n_{1} n_{2}} \\
& +e^{-i \theta} V_{n_{1}-n_{2}}\left(\left(k_{j}-k_{i}\right) e^{-i \theta}\right),
\end{aligned}
$$

where

$$
k_{j}=j \Delta k \quad(j=-m,-m+1, \ldots, 0,1, \ldots, m)
$$

and $N_{0}=2 m+1$ is the total number of grid points. In Eq. (26), the structure of $\hat{H}_{F}$ is best organized by ordering components such that $k_{i}\left(k_{j}\right)$ runs over all grid points before each change in the Floquet block index $n_{1}\left(n_{2}\right)$. This completes the construction of the non-Hermitian Floquet Hamiltonian in the momentum representation. The complex quasienergy eigenvalues and eigenvectors can now be determined by the solution of the non-Hermitian eigenproblem of $\hat{H}_{F}(\theta)$.

\section{INTENSITY-DEPENDENT COMPLEX QUASIENERGIES AND THE STABILITY OF MODEL $\mathrm{Cl}^{-}$IONS IN VERY INTENSE LASER FIELDS}

In this section we present an application of the momentum representation CSFGH method to the study of the complex quasienergies and stability of negative ions in strong fields. As we have suggested in Sec. I, the study of the behavior of negative ions in very intense fields appears feasible in currently available experimental facilities. In accordance with the short-range characteristics and also with the experimental data of the binding energy of the $\mathrm{Cl}^{-}$ion $\left(E_{0}=3.613 \mathrm{eV}\right)$, we choose a one-dimensional short-range model potential of the form [Eq. (12)] to study its behavior in very intense laser fields. Moreover, we consider the light source to be an ArF laser $(193 \mathrm{~nm})$, one of the highest-brightness excimer lasers currently available. Most of these lasers can routinely deliver laser intensities in excess of $10^{17} \mathrm{~W} / \mathrm{cm}^{2}$ in the spot focal region [6]. The uv frequency of the laser light $(\omega=6.424 \mathrm{eV})$ guarantees the high-frequency condition necessary for significant suppression, though it is not asymptotically high.

Figure 1 shows the momentum-space potential functions $V_{n}(k)$ in the $\mathbf{K H}$ frame. [Note that
$V_{-n}(k)=(-1)^{n} V_{n}(k)$.] Figure $1($ a $)$ depicts $V_{0}(k)$ for a range of $\alpha_{0}\left(\equiv e\left|\mathbf{E}_{0}\right| / m \omega^{2}\right)$, where $\omega$ is fixed at $193 \mathrm{~nm}$. Figure (1b) shows the function form of $V_{n}(k)$ for the specific case of $\alpha_{0}=3.0$ a.u. We first keep only the zeroth-order term $V_{0}$ in Eq. (22) and diagonalize the discretized Hamiltonian for different laser intensities. The solid, dashed, and dash-dotted lines in Fig. 2 are the bound-state energies of this field-deformed Hamiltonian. In addition to the ground state (solid line), two new bound states (dashed and dash-dotted lines) appear successively with increasing field strength $\left(\alpha_{0}\right)$. The lowest two states become nearly degenerate in the highest intensities shown. The structure of the negative ion is strongly distorted by the fields.

This structure is further modified by higher-order coupling terms $V_{n}(n \neq 0)$. The three circle curves in Fig. 2 are the real parts of the complex energies of the three
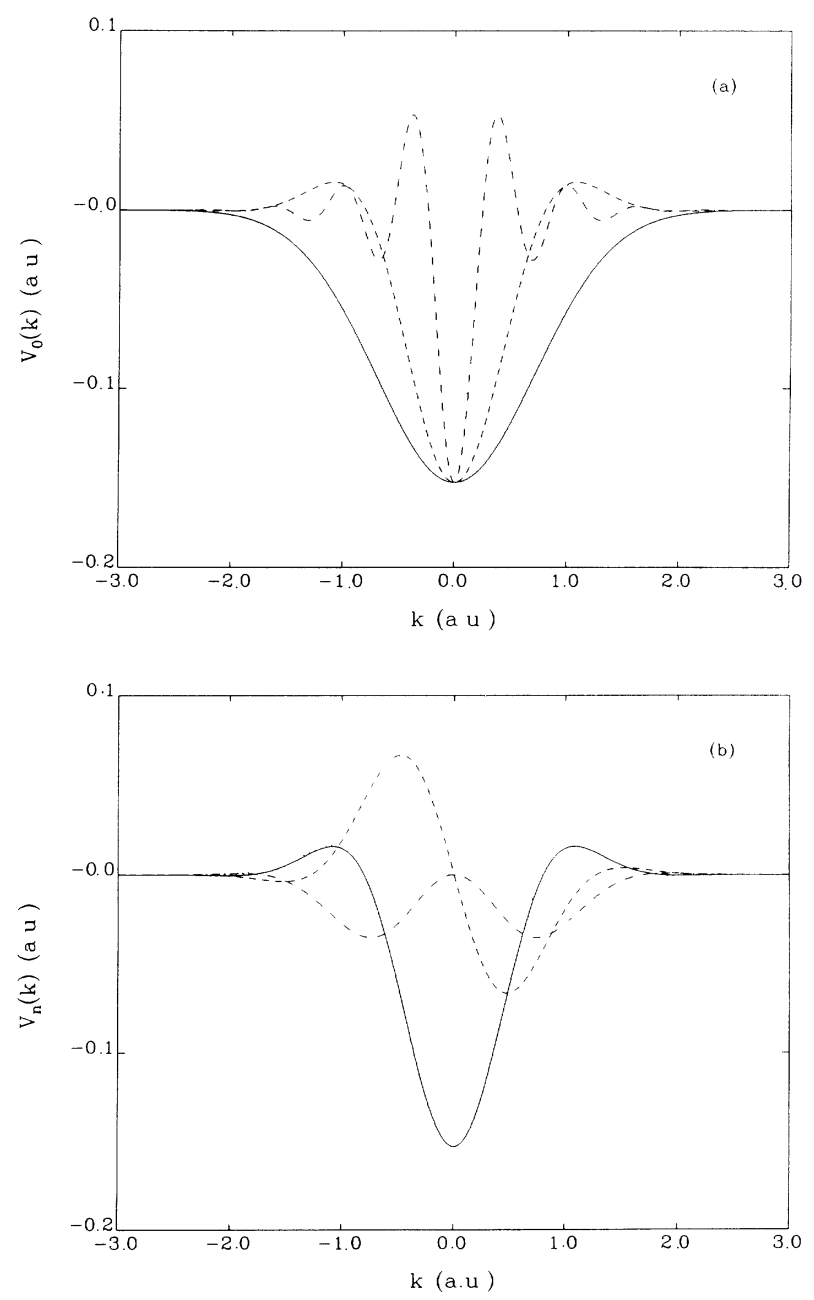

FIG. 1. Momentum-space potential forms in the $\mathbf{K H}$ frame. (a) Zeroth-order potentials $V_{0}(k)$ for different values of $\alpha_{0}$. Solid line, $\alpha_{0}=0.0$ a.u.; dashed line, $\alpha_{0}=3.0$ a.u.; dash-dotted line, $\alpha_{0}=10.0$ a.u. (b) Potentials (or couplings) $V_{n}(k)$ for $\alpha_{0}=3.0$ a.u. and for different orders. Solid line, $n=0$; dashed line, $n=1$; dash-dotted line, $n=2$; dotted line, $n=3$. Unless otherwise indicated, the ArF laser with $\omega=6.424 \mathrm{eV}$ (193 nm) and a model $\mathrm{Cl}^{-}$ion are considered throughout the paper. 


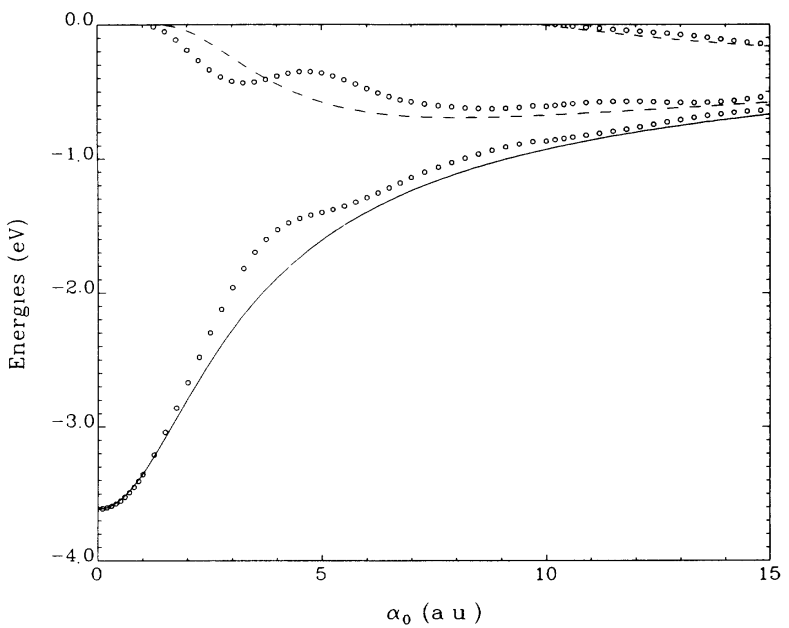

FIG. 2. Energies of the three field-deformed bound states as a function of $\alpha_{0}$. The solid, dashed, and dash-dotted lines correspond to the energies of these states when high-order couplings $V_{n}(n \neq 0)$ are neglected, whereas the circle lines are the corresponding coupled-channel Floquet results (the real parts of the complex quasienergies).

quasienergy states obtained by the solution of the coupled-channel Floquet Hamiltonian, Eq. (26), using the CSFGH method in momentum representation. Up to ten Floquet channel blocks are included in the calculation for the highest field strength under consideration. For each Floquet channel block $(n)$, we used 81 grid points to discretize the $k$ space. All the results shown have converged to at least $10^{-7}$ a.u. The coupled-channel results approach the zeroth-order results in both weak- and high-intensity limits but deviate significantly for moderate field strengths. In particular, for $\alpha_{0} \sim 5.0$, the two lowest states show strong repulsion to each other.

Shown in Fig. 3 are the converged CSFGH results for the photodetachment rates $\Gamma=2|\operatorname{Im}(E)|$ of the model negative $\mathrm{Cl}^{-}$ion at $193 \mathrm{~nm}$ for a range of laser intensities $\left(\alpha_{0}\right)$ from the weak to the very intense regime. Figure 3(a) corresponds to the detachment rate of the ground state and Figs. 3(b) and 3(c) to that of the first and of the second new bound states. While the decay rate $(\Gamma)$ increases rapidly with increasing $\alpha_{0}$ at the beginning, it decreases with further increasing $\alpha_{0}$ after reaching a maximum $\Gamma$ value at $\alpha_{0} \simeq 2.25$ a.u. [Fig. 3(a)]. This phenomenon is often referred to in the literature as the "suppression of photoionization" or "stabilization of atoms" in very intense fields $[1,3,4]$. However, as can be seen here in Fig. 3(a), this "suppression" behavior is by no means monotonic in $\alpha_{0}$. In fact $\Gamma$ reaches a minimum at $\alpha_{0} \simeq 5.0$ a.u., and after that it increases again (in the sense that the ion becomes less stable). Stabilization revives after the rate $(\Gamma)$ reaches the second maximum (at $\alpha_{0} \cong 7.0$ a.u.) and the rate decreases to a higher degree when it reaches the second minimum (at $\alpha_{0} \cong 10.5$ a.u.). This process repeats when we further increase the field strength or $\alpha_{0}$. The other two new bound states exhibit similar oscillatory behaviors [see Figs. 3(b) and 3(c)].
In a recent calculation using the same model potential but different physical parameters (for describing model neutral atoms with a few bound states) and numerical techniques, Bardsley and Comella [11] have also observed nonmonotonic behavior in $\Gamma$. As the data they obtained might not be enough to trace the detailed behavior, and
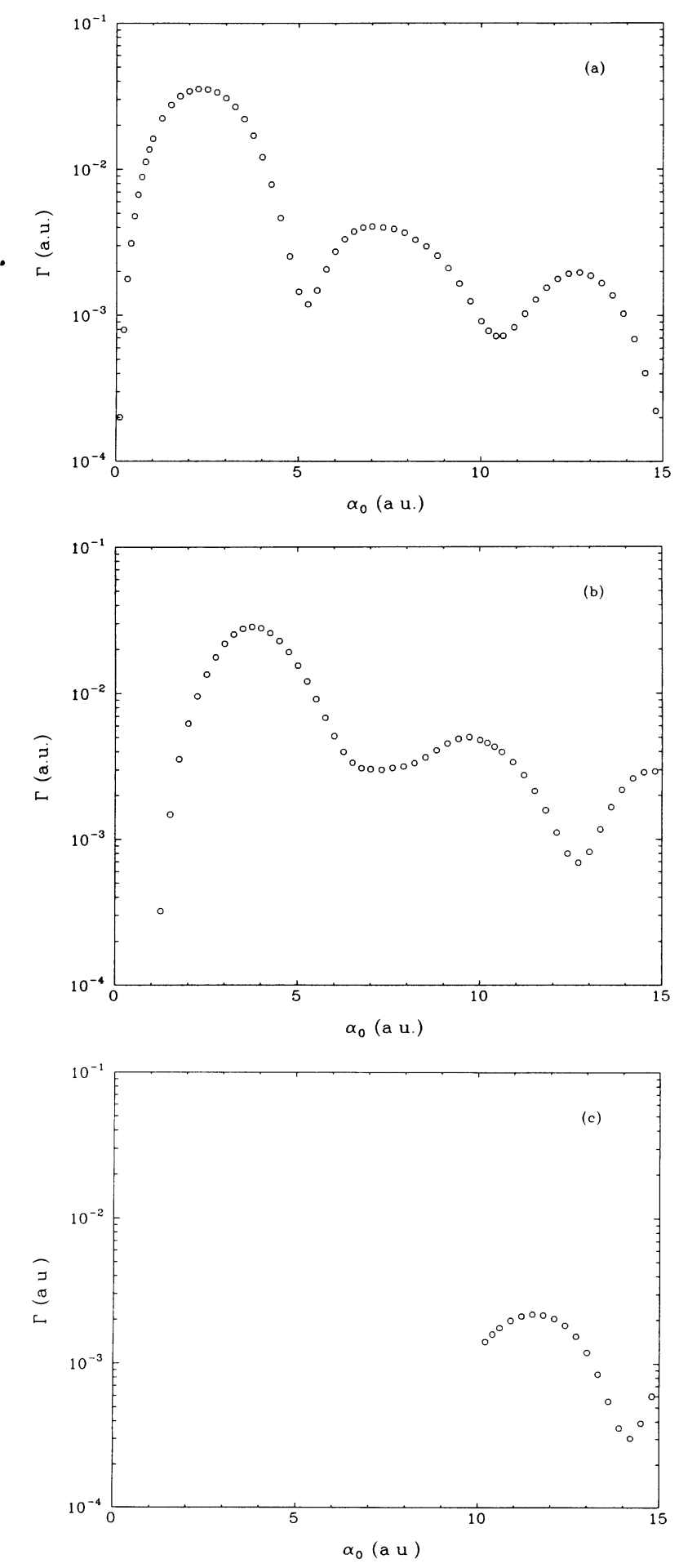

FIG. 3. Converged results for the photodetachment rates (widths $\Gamma$ ) of (a) the ground state, (b) the first new excited state, and (c) the second new excited state, as a function of $\alpha_{0}$. 
also more bound states might complicate the analysis in their calculations, their results exhibit strong fluctuations so that it is hard to draw any conclusion. Moreover, no explanation of the behavior was given.

Here in the present calculations, the oscillatory structures of the photodetachment rates $(\Gamma)$ are very pronounced, although the tendency toward the overall reduction of decay rates is apparent. In contrast to the "death valley" discussed by Pont and Gavrila [3], the minimum regions in Figs. 3(a) -3 (c) are favorable valleys for observing suppression of the ionization of negative ions. This is further explored in the following section.

\section{DISCUSSIONS}

Table I presents the critical values of the oscillatory behavior shown in Fig. 3(a). Note that the maximum intensity considered here is $2.45 \times 10^{16} \mathrm{~W} / \mathrm{cm}^{2}$. It is seen that even at the first maximum $\left(\alpha_{0} \simeq 2.25\right.$ a.u. $)$ where the ion decays fastest, it can still survive more than six optical cycles or longer than $4 \mathrm{fs}$. This is in contrast to the situation considered in Ref. [3] where the shortest lifetime is only one cycle or $0.1 \mathrm{fs}$. In addition, in the higher field strengths, the lifetime of the negative ions can be as long as subpicoseconds, which are within the regime of current laser technology. Of course, quantitative evaluation of the possibility for observing such suppression requires the consideration of the pulse shape. But the rate calculations presented here have already provided insights and shown some prospects. More detailed consideration of the pulse-shape effect is in progress and will be presented elsewhere.

The interesting oscillatory behavior may be qualitatively understood if we analyze the detachment process in the $\mathrm{KH}$ frame. In Eq. (22) it is seen that $V_{0}(k)$ serves as the dressed potential, while $V_{n}(k)$ provides $n$-photon couplings between different states. From Eq. (23), one sees that couplings are determined primarily by the Bessel functions which are oscillatory with respect to the parameter $\alpha_{0}$. Thus the $n$-photon coupling between the field-deformed (zeroth-order) ground and continuum states is $\left\langle\psi_{e}\left|V_{n}\right| \psi_{0}\right\rangle$ and the corresponding decay rate is given approximately by

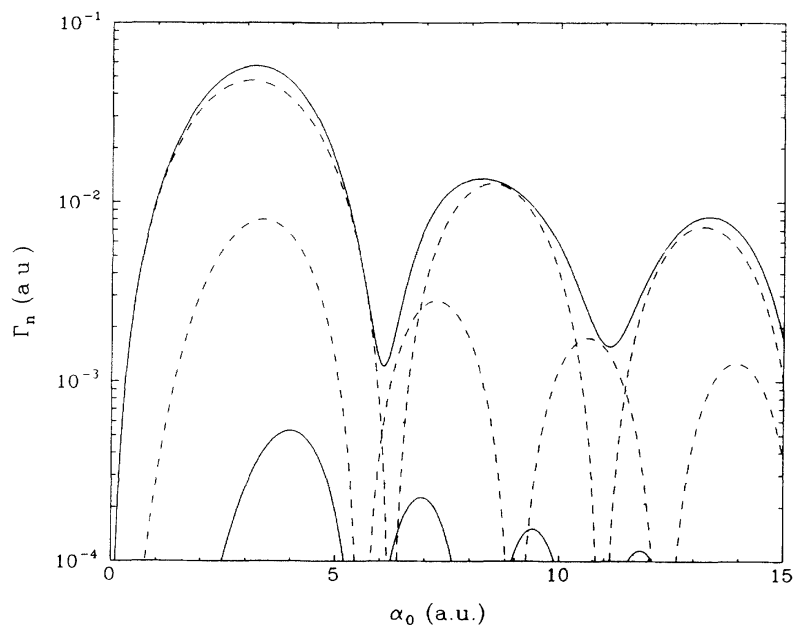

FIG. 4. Detachment rates as a function of $\alpha_{0}$ calculated from Eq. (30). The total detachment rate is given by the topmost solid curve. The partial rates $\Gamma_{n}$ are given, respectively, by the dashed line $(n=1)$, the dash-dotted line $(n=2)$, the dotted line $(n=3)$, and the bottom solid line $(n=4)$.

$$
\Gamma_{n} \cong 2 \pi\left|\left\langle\psi_{E}\left|V_{n}\right| \psi_{0}\right\rangle\right|^{2},
$$

where $\left|\psi_{0}\right\rangle$ and $\left|\psi_{E}\right\rangle$ are, respectively, the ground and continuum states of the zeroth-order Hamiltonian in Eq. (22), and $\left|\psi_{E}\right\rangle$ is energy normalized. In the highfrequency field, even the lowest-energy electron is emitted from the high-lying continuum, so the continuum state may be approximated by the plane wave, i.e.,

$$
\left|\psi_{E}\right\rangle \cong\left(\frac{m}{k_{n}}\right)^{1 / 2}\left|k_{n}\right\rangle .
$$

Consequently, the $n$-photon photodetachment rate is approximately

$$
\Gamma_{n} \cong \frac{2 \pi m}{k_{n}}\left|\int d k V_{n}(k) \psi_{0}\left(k_{n}-k\right)\right|^{2}
$$

where $\psi_{0}(k)$ is the $k$ space wave function of the field-

\begin{tabular}{|c|c|c|c|c|c|}
\hline & $\alpha_{0}$ (a.u.) & $I\left(\mathrm{~W} / \mathrm{cm}^{2}\right)$ & $\Gamma^{a}$ (a.u.) & $\tau^{\mathrm{b}}$ (opt. cyc) & $\tau^{\mathrm{c}}(\mathrm{fs})$ \\
\hline $\begin{array}{c}\text { maxt } \\
\max \end{array}$ & 2.25 & $5.51 \times 10^{14}$ & $3.5368 \times 10^{-2}$ & 6.7 & 4.2 \\
\hline $\begin{array}{l}\text { 1st } \\
\text { min }\end{array}$ & 5.25 & $3.00 \times 10^{15}$ & $1.1891 \times 10^{-3}$ & 198.5 & 127.8 \\
\hline $\begin{array}{l}\text { 2nd } \\
\max \end{array}$ & 7.0 & $5.33 \times 10^{15}$ & $4.0661 \times 10^{-3}$ & 58.1 & 37.4 \\
\hline $\begin{array}{l}\text { 2nd } \\
\text { min }\end{array}$ & 10.4 & $1.18 \times 10^{16}$ & $7.2380 \times 10^{-4}$ & 326.2 & 209.9 \\
\hline $\begin{array}{l}3 \mathrm{rd} \\
\max \end{array}$ & 12.7 & $1.76 \times 10^{16}$ & $1.9716 \times 10^{-3}$ & 119.7 & 77.1 \\
\hline
\end{tabular}

TABLE I. Critical lifetimes and photodetachment rates of the ground state of the model $\mathrm{Cl}^{-}$ion in very intense laser fields at $193 \mathrm{~nm}$.

${ }^{a} \Gamma$ is the total ionization width (rate) in atomic units.

${ }^{\mathrm{b}} \tau$ is the lifetime in terms of the number of field oscillations.

${ }^{c} \tau$ is the lifetime in units of femtoseconds. 
deformed ground state. Since the ground-state wave function always dominates at $k=0$, we approximate the integral by taking the dominant contribution of $\psi_{0}(k)$, i.e., by taking the integrand at $k=k_{n}$. This gives
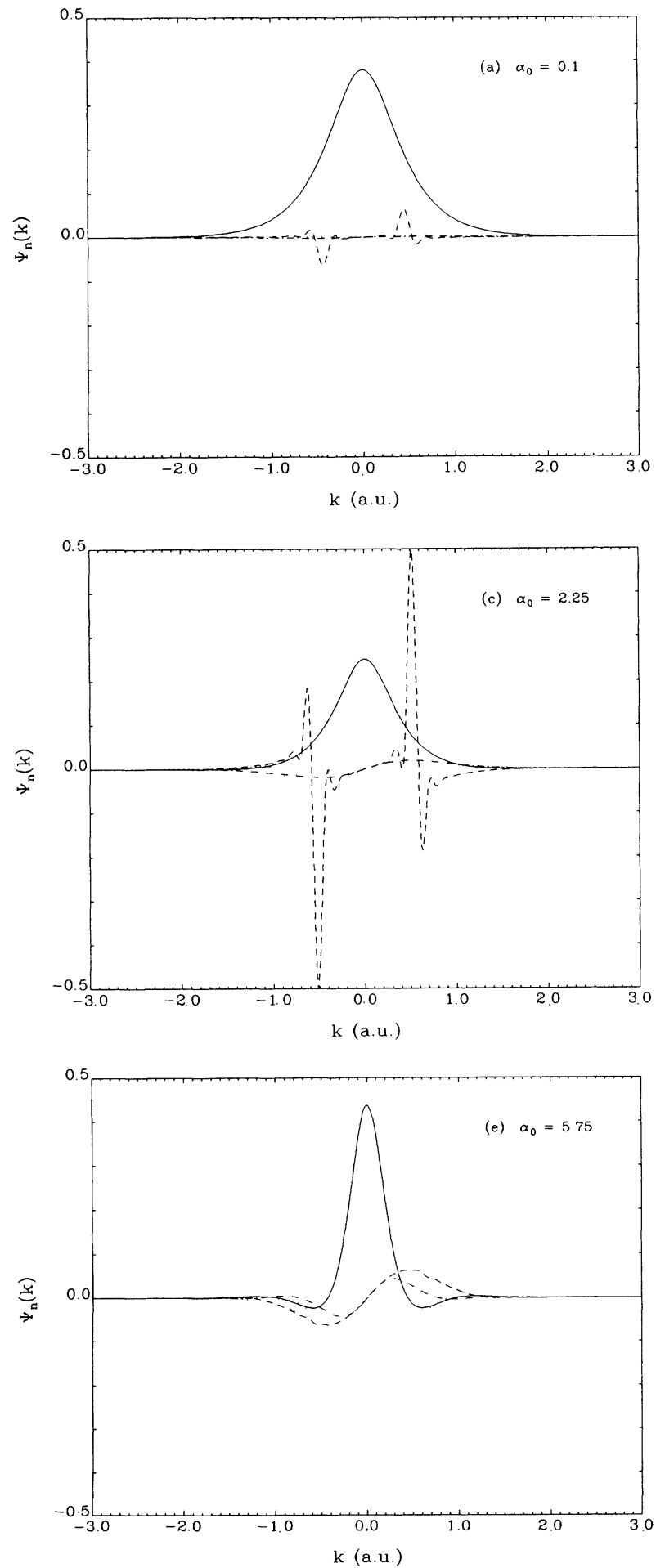

$$
\Gamma_{n} \approx \frac{2 \pi}{k_{n}}\left|V\left(k_{n}\right)\right|^{2}\left|J_{n}\left(\alpha_{0} k_{n}\right)\right|^{2},
$$

where $k_{n}=\sqrt{2 m\left(n \omega-E_{0}\right)}$ is the momentum of the electron liberated by absorption of $n$ photons and $E_{0}$ is the
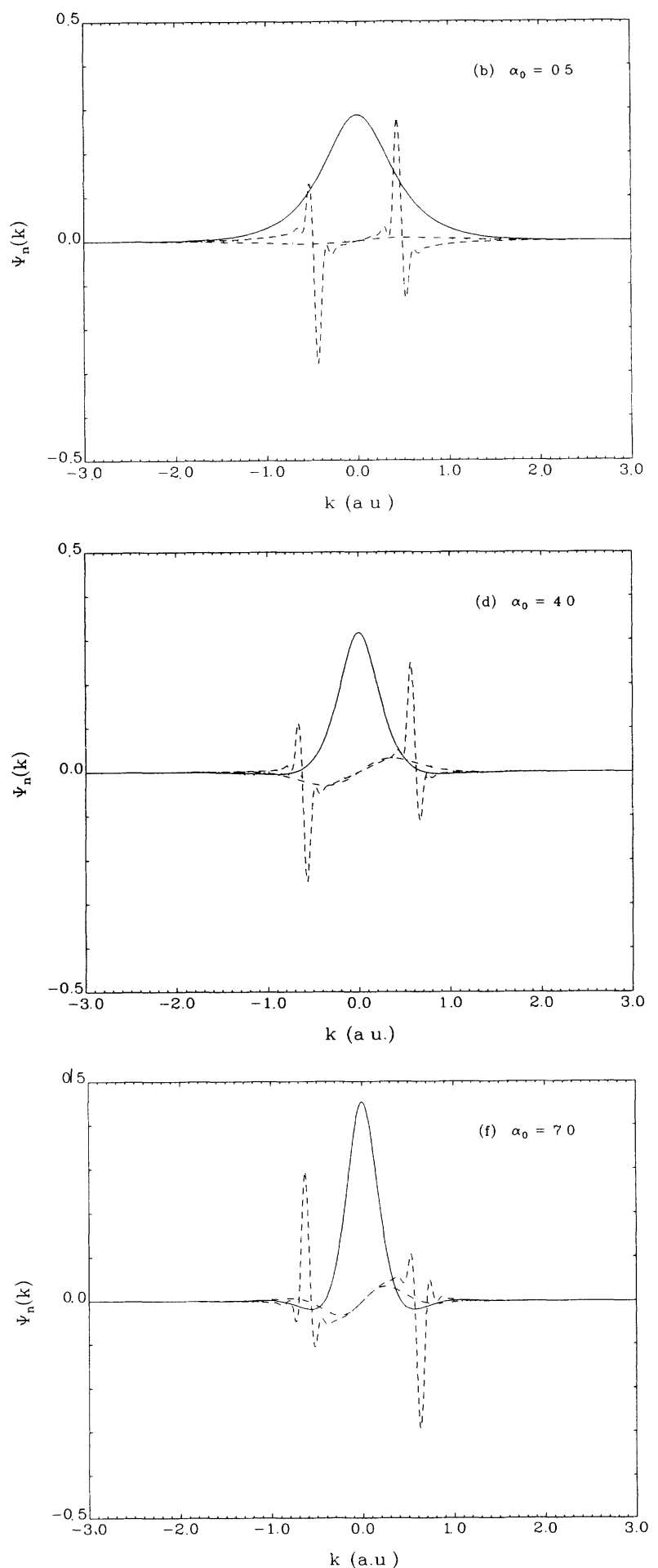

FIG. 5. Momentum-space wave functions of the quasienergy state correlated with the ground state of the model $\mathrm{Cl}^{-}$ion for different values of $\alpha_{0}$. Solid, dashed, dotted, and dash-dotted lines are, respectively, components for photon orders $n=0,-1$ (absorption of one photon), -2 (absorption of two photons), and 1 (energy nonconserving component). Note the oscillatory behavior of the amplitude of the $n=-1$ component with increasing $\alpha_{0}$. (a) $\alpha_{0}=0.1$ a.u., (b) $\alpha_{0}=0.5$ a.u., (c) $\alpha_{0}=2.25$ a.u., (d) $\alpha_{0}=4.0$ a.u., (e) $\alpha_{0}=5.75$ a.u., (f) $\alpha_{0}=7.0$ a.u. 
(field-deformed) initial bound-state energy.

Figure 4 displays the partial decay rates $\Gamma_{n}(n=1,2,3,4)$ and the total decay rate (the topmost solid line) obtained approximately by $\Gamma=\Sigma_{n} \Gamma_{n}$ of the ground state as a function of $\alpha_{0}$. In spite of the approximations made in Eqs. (27)-(30), Fig. 4 reproduces all the qualitative features of the exact results shown in Fig. 3(a). It is remarkable that the maxima and minima and therefore the oscillatory behavior in photodetachment rates are determined primarily by the Bessel functions, a salient feature of the nonlinear response of negative ions in very intense fields. We note that while Pont and Gavrila [3(a)] have also used (similar but not identical) expressions involving Bessel functions, no oscillatory behavior was reported in their results of $\Gamma$. The origin for this difference in the behavior of $\Gamma$ is not precisely known. However, there are significant differences between the potential forms used in their and our calculations and some possible explanations are given below. (i) As was pointed out by Pont [3(c)], the partial rates drop with roughly the square of the number of photons absorbed. For the Gaussian potential, however, it is seen from Fig. 4 that the partial rates $\Gamma_{n}$ drop much more quickly than $n^{-2}$. Figure 4 also shows different $\Gamma_{n}$ oscillatory behavior for different photon number $n$. The near-exponential drop of $\Gamma_{n}$ with respect to the number of photons absorbed results in the dominance of the single-photon decay rate $\Gamma_{1}$ for most $\alpha_{0}$. Thus the oscillatory behavior of the total decay rate for the present Gaussian potential can be approximately accounted for by the Bessel function of order 1. On the other hand, there are more photon absorption channels contributed to the Coulomb potential case. Hence, the oscillatory behavior in the total decay rate could be averaged out and masked for atomic hydrogen. (ii) The total decay rate obtained by integration of the angular decay rates over all directions may account for another possible source that could mask the oscillatory behavior in the 3D Coulomb potential case. The angular decay rates [see, for example, Eq. (8) of Ref. [3(a)] and Eq. (61) of Ref. [3(b)]] are always oscillatory as a function of $\alpha_{0}$, but the total decay rate is not necessarily oscillatory. This is not the case for the present 1D Gaussian potential, where the angular distribution is not considered. A detailed study of the angular distribution behavior of $3 \mathrm{D}$ short-range model negative ions is needed before a conclusion can be reached [14].

While qualitative features can be interpreted by this simple argument, quantitative prediction of the photodetachment rates requires the nonperturbative solution of the coupled-channel Floquet CSFGH calculation, such as we have performed in Sec. III. It is useful at this point to summarize the main results from the above analysis: (i) the conventional regime where the lifetime of the atom decreases with increasing field intensity is simply the rising part of the Bessel functions, (ii) the suppression regime of current interest corresponds to the falling part of the Bessel functions, and (iii) the oscillatory behavior of the decay rates also stems from the oscillatory properties of the Bessel function and we might be tempted to define an additional regime [15].
It is also instructive to examine the behavior of corresponding quasienergy eigenfunctions in momentum space. For simplicity, we show only the real quasienergy eigenfunctions obtained by the coupled-channel Floquet FGH calculations without the use of complex scaling (i.e., $\theta=0$.) Figures 5(a) $-(5(f)$ display such eigenfunctions for different photon indices $(n)$ and for various field strengths $\left(\alpha_{0}\right)$. For each graph, the solid, dashed, and dotted lines correspond, respectively, to the photon index $n=0,-1$, and -2 . The dash-dotted line is the eigenfunction component corresponding to the energynonconserving term $(n=1)$. Our focus here is on the $n \neq 0$ components. For $\alpha_{0}=0.1$ a.u., only the $n=-1$ component is appreciable and the high-order components can be treated perturbatively. The amplitude of the $n=-1$ eigenfunction component increases with increasing $\alpha_{0}$ until it reaches its first maximum for $\alpha_{0}=2.25$ a.u., which is consistent with the rising part in Fig. 3(a). It is interesting to see that with further increasing of the field strength $\left(\alpha_{0}=4.0\right.$ a.u.) this component is in fact reduced, and the $n=-2$ component becomes comparable. In particular, for $\alpha_{0}=5.75$ a.u., the $n=-2$ component seems to be dominant. Again this corresponds to the first falling part of Fig. 3(a), i.e., the part which is called the suppression region. In accordance with the second rising part in Fig. 3, the $n=-1$ amplitude becomes dominant again for $\alpha_{0}=7.0$ a.u. Similar information can be deduced by transforming the $k$-space quasienergy eigenfunctions into the $x$ space (not shown).

\section{CONCLUSIONS}

In summary, accurate and efficient calculations using CSFGH methods indicate that the photodetachment rates of negative ions in very intense laser fields exhibit oscillatory behavior. The investigations of quasienergy eigenfunctions in both momentum and coordinate spaces confirm and also help us to understand these features. Simple interpretation of the oscillatory structures is presented, which attributes the structures to the characteristic couplings between field-deformed bound and continuum states in very intense laser fields. Our onedimensional model study in this regime suggests that negative ions may be the more suitable candidates (than neutral atoms) for the experimental observation of ionization suppression in very intense fields. A more detailed study of this problem is in progress, including threedimensional calculations of angular distributions and the consideration of pulse-shape effects.

\section{ACKNOWLEDGMENTS}

This work was partially supported by the Department of Energy, Division of Chemical Sciences. Acknowledgment is also made to CRAY Research Inc. for the access to CRAY-YMP supercomputer facilities at NCAR (Boulder, CO). 
[1] (a) M. Pont, N. Walet, M. Gavrila, and C. W. McCurdy, Phys. Rev. Lett. 61, 939 (1988); (b) M. Pont. N. Walet, and M. Gavrila, Phys. Rev. A 41, 477 (1990); (c) M. Gavrila and J. Z. Kaminski, Phys. Rev. Lett. 52, 613 (1984).

[2] W. C. Henneberger, Phys. Rev. Lett. 21, 838 (1968); H. A. Kramers, Collected Scientific Papers (North-Holland, Amsterdam, 1956), p. 272.

[3] (a) M. Pont and M. Gavrila, Phys. Rev. Lett. 65, 2362 (1990); (b) M. Pont, Phys. Rev. A 44, 2141 (1991); (c) M. Pont, Phys. Rev. A 44, 2152 (1991).

[4] See, for example, (a) X. Tang and S. Basile, Phys. Rev. A 44, R1454 (1991); (b) K. Burnett, P. L. Knight, B. R. Piraux, and V. C. Reed, Phys. Rev. Lett. 66, 301 (1991); (c) K. C. Kulander, K. J. Schafer, and J. L. Krause, ibid. 66, 2601 (1991); (d) Q. Su, J. H. Eberly, and J. Javanainen, ibid. 64, 862 (1990).

[5] M. Dörr, R. M. Potvliege, D. Proulx, and R. Shakeshaft, Phys. Rev. A 43, 3729 (1991).

[6] C. K. Rhodes, Science 229, 1345 (1985); see also, for example, paper no. JMA1-JMJ4, in Technical Digest of International Conferece of Quantum Electronics, 1990.
[7] S. I. Chu, Chem. Phys. Lett. 167, 155 (1990).

[8] S. I. Chu, J. Chem. Phys. 94, 7901 (1991).

[9] C. C. Marston and G. G. Balint-Kurti, J. Chem. Phys. 91, 3571 (1989).

[10] See, for example, X. Tang and R. Shakeshaft, Z. Phys. D 5, 27 (1987); J. N. Bardsley, A. Szöke, and M. J. Comella, J. Phys. B 21, 3899 (1988).

[11] J. N. Bardsley and M. J. Comella, Phys. Rev. A 39, 2252 (1989).

[12] T. S. Ho, S. I. Chu, and J. V. Tietz, Chem. Phys. Lett. 96, 4664 (1983); T. S. Ho and S. I. Chu, J. Phys. B 17, 2101 (1984).

[13] For reviews of complex-scaling Floquet methods, see S. I. Chu, Adv. At. Mol. Phys. 21, 197 (1985); S. I. Chu, Adv. Chem. Phys. 73, 739 (1989).

[14] G. Yao and S. I. Chu (unpublished).

[15] The behaviors (i) and (ii) summarized in this paragraph may be gleaned from the results of Ref. [3]. However, the behavior (iii) reported here has not been previously discussed. 